\title{
Escape from the Computer Lab: Education in Mobile Wireless Networks
}

\author{
Elizabeth M. Belding-Royer \\ Department of Computer Science \\ University of California, Santa Barbara \\ Santa Barbara, CA 93106 \\ ebelding@cs.ucsb.edu
}

\begin{abstract}
As mobile wireless network technology becomes widespread, the importance of education about this new form of communication is becoming critical. One of the benefits of mobile computing education is that courses focusing on this topic can leverage advances in wireless technology to support novel education and learning paradigms. In particular, the development of wireless communication, together with portable devices, allows the freedom of both mobility and creativity in curriculum and assignments. This paper describes two recently designed mobile computing courses at the University of California, Santa Barbara, focusing on the methodologies for incorporating handheld computing devices into the course assignments. Course evaluations have shown that the use of mobile computing devices has been well-received by the students in both of these courses, and we encourage their incorporation into similar courses.
\end{abstract}

\section{Introduction}

Advances in technology have enabled new education and learning paradigms. The introduction of new platforms and devices allows non-traditional teaching methodologies that break away from conventional textbook and programming assignments typical of many Computer Science courses. In particular, the advent of wireless communication, together with portable devices, allows the freedom of both mobility and creativity in the course projects and assignments [5, 7].

As research in mobile wireless networking becomes increasingly popular, a number of universities have introduced courses on this topic to prepare students for employment or continued research in this area. These courses range in focus from physical layer characteristics and radio propagation, to networking oriented courses that emphasize the higher layers of the protocol stack. In particular, at the University of California, Santa Barbara (UCSB) we have introduced two new mobile computing courses; one undergraduate and one graduate course.

In designing these two courses, we have taken the opportunity to introduce non-traditional learning methodologies through the incorporation of HP iPAQ handheld computers. iPAQ computers are well-suited for classroom use because of their versatility; they support the Linux operating system and can run programs written in a variety of languages. The iPAQs have been utilized in both mobile networking courses at UCSB, although they have served different purposes in each course. In the undergraduate course they are used for the completion of homework assignments, while in the graduate course they are used as an option for completing a class project. In both cases, the feedback on the inclusion of these devices has been positive; students enjoy both getting out of the computer lab to complete assignments and gaining experience with new platforms.

The goal of this paper is to report on the experiences we have had in using the iPAQs in our mobile networking courses. We describe the objectives of the courses and the assignments for which the iPAQs were utilized. We also discuss both the positive aspects and difficulties we have had in the inclusion of these devices so the other educators can successfully integrate the devices into their course. Based on the feedback from the students, we strongly highly recommend the incorporation of handheld computing devices into mobile computing, as well as other, courses.

\section{Course Objectives}

The objectives of the undergraduate and graduate courses differ slightly due to their different emphases, attendees, and placement in relation to other courses. The undergraduate course is the third course in our three part undergraduate networking series. The first networking course is an introduction to the protocol stack, while 
the second course is a network programming course. The third course, which was introduced to the series in the 2001-2002 academic year, is an advanced topics course. The first half of the course is intended to be a survey of advanced topics, such as multicast and quality of service, while the second half is an in-depth study of one particular topic. For the first two years of the course offering, the topic has been mobile networking. Because this course is the third in a three part series, by the time the students take this course they have a solid background in networking and have experience with network programming. Hence this course offers a good opportunity for more intriguing homework assignments. Students are expected to finish this course with an enriched understanding of networking and a deeper understanding of the particular focus of the course. In the first two years of the course offering, there have been about 35 students enrolled in each class.

At the graduate level, students taking the Mobile Computing course are expected to have previously taken the graduate level advanced networking course, although this is not explicitly required. At a minimum, however, students must have a solid undergraduate understanding of networking. The objective of this course is to give students an indepth study of mobile networks and mobile computing systems. The class typically has about 40 students, many of which are likely to pursue networking, either mobile or wired, as their graduate thesis topic. The project-oriented nature of the course allows students to develop and test their own ideas to improve mobile networks and computing systems. Students have the choice of utilizing either simulation or implementation for their project. If the student selects an implementation experiment, which is based on iPAQs, then s/he has the option to test or leverage existing software if an original use of that software is proposed. Alternatively, the student is free to develop new software for the iPAQ.

For both the undergraduate and graduate courses, students that successfully complete the 11-week course acquire a deep understanding of the characteristics of mobile networks that set them apart from wired networks and the solutions that are utilized to make these networks function. Through the use of the iPAQs, the students obtain first-hand experience with the ephemeral nature of wireless connectivity, as well as with the uniqueness and freedom of movement intrinsic to wireless networks.

\section{Requirements}

In terms of hardware, the basic necessities include iPAQ handheld computers, and wireless network interface cards (NICs), serial cables and power adapters for each handheld. The serial cables are needed so that Linux can be loaded onto the iPAQs. While it is optimal to have an iPAQ for each student, the class at UCSB is popular enough that we have never have enough iPAQs for a one-to-one pairing. To ensure that each student has a fair amount of time with the iPAQ, we typically ask the students to establish groups of two, and we give each pair an iPAQ. However, the students are not allowed to work together on the assignment; they must simply share the hardware. Because a portion of the assignments can be completed without the iPAQ, this arrangement has so far worked well.

The iPAQs we have traditionally used are model 3670s. They have $206 \mathrm{MHz}$ StrongArm processors with $64 \mathrm{MB}$ RAM. While they are not the latest model, they have had sufficient capabilities for the assignments. However, occasionally the students have complained about the lack of storage and have been forced to minimize the amount of information they store on the iPAQ itself. Compact flash (or other PCMCIA adaptable storage technologies) can be used to supplement the internal memory; however, we have not yet utilized this solution.

The wireless NICs we use are PCMCIA Lucent Orinoco Silver 802.11 b cards. These cards have worked well and we have had no difficulty using them with the iPAQs. In addition to the Orinocos, we have also tried Avaya wireless NICs. These cards resulted in some compatibility problems when used with the double sleeves for the iPAQs. As a consequence, we now use only the Orinoco cards.

On the network end, an infrastructured wireless network with access points is required for connectivity to the remainder of the network. Each access point must have a corresponding wireless NIC. The access points used throughout our department are Lucent Orinoco AP-1000's.

\section{Assignments}

The objective of the undergraduate assignments is threefold. The first objective is to introduce students to the dynamic nature of wireless connectivity by demonstrating the variability of signal strength. The second objective is to reinforce concepts covered during the course lectures. In particular, wireless characteristics, access point handoffs and Mobile IP [6] operation are emphasized. The third major objective is to introduce and acclimate students to a new computing platform. Most students enrolled in the course are primarily accustomed to working with workstations and PCs. A fourth and more minor objective is to give the students experience with ad hoc/peer-to-peer network connectivity.

In order to begin the assignments, the students must first install a clean installation of the Linux operating system on the iPAQs. We utilize the Familiar Linux distribution [1], which is designed specifically for iPAQ devices. We have found that a fresh install of Linux at the beginning 


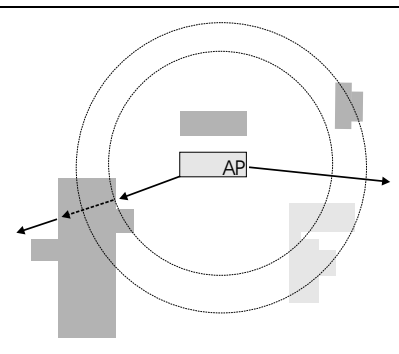

\section{Figure 1. Access Point and Measurement Directions.}

of the quarter not only assures us that we are using the latest Familiar release, but also eases problem solving later on by eliminating any misconfigurations or other errors introduced by the previous iPAQ user.

\section{Signal Strength and Link Quality}

The first homework assignment focuses on the first objective. In this assignment, students perform repeated measurements of signal strength from a specific access point on campus. They must first develop server and client applications so that they can stream constant bit rate (CBR) traffic from some node on the wired network (the server) to the handheld (the client). This introduces the students to code development for the iPAQ. The skiffcluster [2] is an invaluable resource in the development of the client because it allows the students to remotely compile their code in a native StrongARM environment, which is more user-friendly than the iPAQ. Once compiled on the cluster, the executable can then be downloaded directly to the iPAQ. In addition to the skiffcluster, cross compilers are also available and can be utilized to compile kernel modules. While not needed for this assignment, the cross compilers are essential for some of the graduate student projects (see section 6).

In this first assignment, students measure signal strength for both uni-directional and bi-directional connectivity to the access point at specific distances and in specific directions from the access point. They must also compute the average error rate at each of these locations. Figure 1 illustrates the location of the access point and the directions in which the signal strength must be measured. Each measurement must be performed twice at different times on the same day. The measurements must then be repeated on a second day. Hence, the students take each measurement a total of four times. They then take the same measurements, this time communicating directly with another student, instead of the access point.

Through this introductory assignment, the students learn one of the fundamental characteristics of wireless communication: that it is unpredictable and signal quality is based on a number of factors, not only distance. For instance, by taking the measurements on different days and at different times of the days, the students learn that the quality of the signal is impacted by the weather and the exterior temperature. Inadvertently, they also learn that the measurement is sensitive to orientation of the device, as well as obstacles. Students have frequently noted that if they take the measurement between classes, the signal quality experiences more fluctuations as other students pass between the iPAQ and the access point. As shown in the figure, the students take the measurement in different directions from the access point. In one case there is a line-of-sight path, while in the other there is a building directly between the iPAQ and the access point. Because this building is fairly obstructing, there is little, if any, signal received on the opposite side of the building. Hence, the impact of obstacles on signal propagation is learned.

\section{Handoffs}

The objective of the second homework assignment is to introduce students to handoffs and help them understand why and when handoffs occur, as well as the effect of handoffs on data packet transmission.

Wireless access points are deployed on the first three floors of Engineering 1, the building in which the Computer Science department at UCSB is housed. Students are given a map of the location of these access points and are told to walk down the hall on the second and third floors and mark the location of each handoff that occurs. In addition to marking the location of the handoffs, the students must also note the address of the access points they hand off between, as well as the signal quality metric before and after the handoff. The signal quality can be obtained through the iwconfig utility.

Once they have determined the handoff locations, students must then pick a location and measure the performance impact of the handoff. They use their client/server application to stream data from the server to client. The streaming rate and the data packet size are pre-defined and vary by experiment. For each data packet size/streaming rate combination, the student must measure the packet loss during the handoff, and from the measurement, calculate the handoff latency.

The final part of this assignment is to plot the data packet reception by indicating the sequence number of each packet received. This aids in determining the handoff latency, since packets can be lost throughout the experiment, not only during the handoff. From this graph, the students can visualize exactly when the handoff occurs.

This assignment helps students to further understand the nature of wireless signal propagation. The students learn that they often receive signals from multiple access points, 
and that sometimes an access point located on a floor below or above them provides the strongest signal. By comparing their results with other students, the unpredictability of wireless communication is further emphasized because the handoff locations, signal strengths, and access point to which they handoff often varies between students. The variation in streaming rates enables students to obtain different granularities in handoff latency measurements, and shows them that the latency is typically fairly insignificant since few packets are dropped.

\section{Mobile IP}

In this assignment, the students again perform multiple handoffs between access points; this time the access points are located on different subnets so that students can observe the impact of handing off between subnets. One subnet has a Mobile IP [6] home agent while the other has a Mobile IP foreign agent. The students must install the Mobile IP client on their iPAQ. The students then assign themselves an IP address on the home subnet. It is very important that this IP address is manually assigned and DHCP is not used; otherwise, if DHCP is utilized, the address will not be retained after the handoff to the different subnet. Once the IP address is assigned, connectivity with the Home Agent is established, and streaming data packets are received on the home subnet, a handoff to the second subnet is performed. Hence, the student must be in transmission range of both subnets during the experiment. The student then measures the number of packet losses that occur during the handoff, and from that calculates the handoff latency. The handoff has completed once data packets are again received on the new subnet.

The second part of this assignment is to use traceroute to determine whether route optimization is used as part of the Mobile IP installation. The students initialize on the home subnet and then handoff to the foreign subnet. Once on the foreign subnet, they perform a traceroute from a machine on the Computer Science network (a third, separate subnet) to the iPAQ. From this traceroute, they should be able to discern whether routing optimization is utilized.

As stated previously, the goal of this assignment is to help students understand the extra latency caused by Mobile IP and handing off between subnets. Further, it assists in understanding of Mobile IP operation, because the students must draw a network topography based on the results of their traceroute. They complete the assignment with a much clearer understanding of Mobile IP than they gain through the lecture alone.

\section{Discussion}

\subsection{Potential Pitfalls}

A number of potential problems can arise with the hardware requirements for the course. Because the iPAQs are portable and are in the possession of the students throughout the quarter, it is possible for them to be improperly cared for; they are often subject to a tumultuous duration in the backpack of a student. As a consequence, the devices may malfunction in unpredictable ways. While this limits the future utility of the device, the more immediate consequence is that the $\mathrm{PAQ}$ exhibits unpredictable behavior. For instance, the iPAQ may not properly connect to the wireless network. The most common problem of the iPAQ is that the battery stops holding a charge. While a completely drained battery renders the iPAQ unusable since portability is required to complete the assignment, a different problem arises when the battery holds some charge but drains quickly. When the battery charge is low, the wireless connectivity of the iPAQ becomes unpredictable. It then becomes particularly difficult for students to determine the cause of poor network performance. The impact of this problem can be limited by running some basic operational tests when students first complain of the problem.

An additional hardware problem comes from the reliance on the skiffcluster. While the cluster is fairly reliable, it is somewhat inevitable that connectivity to the cluster, or the cluster itself, will go down the night before an assignment is due. Because this has happened multiple times, we now have a policy for handling assignments that are late because of this problem.

The second set of difficulties stems from the nature of the wireless communication medium. Because wireless communication and signal quality is unpredictable, there is no single "right answer" to the majority of the homework assignments. Although most students typically obtain results within some range of values, occasionally some measurements are significant outliers to the norm. In these situations, it is difficult to determine whether students did the assignment correctly. In most cases, however, we have found that the result variance is usually due to problems with the student code or testing, and once corrected the results are much closer to the median. In a few cases, however, the assignment was done correctly and the quality of the wireless signal was atypical when the measurements were taken. To minimize the occurrence of this problem, the assignments usually require multiple measurements for each data point.

\subsection{Unexpected Benefits}

The assignments have resulted in a number of benefits, many of which were unexpected. The most unexpected ben- 
efit was the publicity of wireless networking that came as a result of others seeing the students in the hallways and on campus with their wireless devices. Multiple faculty asked about the project and about the course in general. On the student side, many students not in the mobile networking course asked where they could obtain these wireless devices and how they could gain access to the wireless network. Many had been previously unaware about the nature and flexibility of wireless communication. This served as significant "free publicity" for general wireless communication.

In addition to the publicity, the other primary benefit of the assignments was the enjoyment of the assignments by the students in the course. The students appreciate the nontraditional nature of the assignments in that they got to work on their homework outside of the computer lab and get some "fresh air." The students also appreciated the "hands-on" nature of the assignments. They expressed that actual experiments with the connectivity enabled a much deeper understanding of its capabilities than simply studying it from a textbook or other materials.

\section{Graduate Course Overview}

The structure of the mobile networking graduate course differs significantly from the undergraduate course. While the undergraduate course is fairly structured in its homework and all students complete the same assignments, in the graduate class students complete projects based on mobile computing. The students can select either a simulation or implementation-based project. The implementation projects typically utilize iPAQs, although the students are free to use their own laptops as well. To successfully complete an implementation-based project, the students can either leverage existing software implementations or they can develop their own. To date, the majority of implementation projects have focused on the network layer or the application layer.

At the network layer, students have taken existing protocol software and first tried to compile and run the software on the iPAQs, and second, tried to obtain some meaningful measurements utilizing this software. Part of the challenge of the network layer implementation projects is in compiling the software and running it successfully on the iPAQs. Software that is not written specifically for the iPAQ architecture does not always execute properly on the devices. As an example, the kernel modules required to run some of the routing protocols must be cross compiled for the ARM architecture. Once the software is successfully installed, the students utilize it to obtain measurements. For instance, in one project the students compared an implementation of the AODV routing protocol [3] with that of the OLSR routing protocol [4] and evaluated a variety of metrics, including throughput and route discovery latency. In another project, the students compared the performance of two different Mobile IP implementations.

The application layer projects have resulted in a number of unique applications developed specifically for the iPAQs. In one project, students developed a virtual business card application, while in another project, students utilized iPAQs with handheld GPS devices and created a coverage map of UCSB's wireless network. Finally, a third project example is a billing system students developed for users of infrastructured wireless networks. The billing client resided on the iPAQ and corresponded with the billing server on the wired network.

\section{Conclusion}

Incorporation of iPAQs into mobile computing courses has resulted in many benefits, both expected and unexpected. Hands-on use of wireless devices enables students to experience first hand the unpredictable nature of wireless connectivity. Because students have indicated that the use of the handheld devices significantly reinforced concepts introduced during class, and because the iPAQs captured student attention more so than traditional computing platforms, we highly recommend the incorporation of handheld computing devices into mobile computing courses, as well as other courses in which they are beneficial. Our plans at UCSB include continued refinement of our mobile computing courses, including the development of additional assignments incorporating handheld computers, as well as the integration of iPAQs into other, non-networking, courses.

\section{References}

[1] The Familiar Project. http://familiar.handhelds.org.

[2] The Skiff Cluster. http://www.handhelds.org/projects/skiffcluster.html.

[3] Elizabeth M. Belding-Royer and Charles E. Perkins. Evolution and Future Directions of the Ad hoc On-Demand Distance Vector Routing Protocol. Ad hoc Networks Journal, 1(1):125-150, 2003.

[4] T. Clausen, P. Jacquet, A. Laouiti, P. Muhlethaler, A. Qayyum, and L. Viennot. Optimized Link State Routing Protocol. In Proceedings of IEEE INMIC, Lahore, Pakistan, December 2001.

[5] Mathias Kolsch, Kristian Kvilekval, and Kevin Almeroth. Improving Speaker Training with Interactive Lectures. In Proceedings of ED-MEDIA, Honolulu, HI, June 2003.

[6] Charles E. Perkins. Mobile-IP, Ad-Hoc Networking, and Nomadicity. In Proceedings of COMPSAC, 1996.

[7] Sami Rollins and Kevin Almeroth. Deploying an Infrastructure for Technologically Enhanced Learning. In Proceedings of ED-MEDIA, Denver, CO, June 2002. 